\title{
Soy Isoflavones
}

National Cancer Institute

\section{Source}

National Cancer Institute. Soy Isoflavones. NCI Thesaurus. Code C65152.

A dietary supplement isolated from soybeans containing phytoestrogen isoflavones.

Although the mechanism of action is unclear, soy isoflavones mimic estrogen action

mediated through estrogen receptors. In addition, this agent also modulates estrogen

metabolism. As a result, soy isoflavones have been shown to reduce tumor cell

proliferation and induce tumor cell apoptosis, as well as to be able to regulate hormone

balance and reduce the risks of breast cancer, heart disease, and osteoporosis. 